\title{
Removal of Common Warts by High-Intensity Focused Ultrasound: An Introductory Observation
}

\author{
Torsten Bove ${ }^{a}$ Tomasz Zawada ${ }^{a} \quad$ Alexander Jessen ${ }^{a} \quad$ Mattia Poli ${ }^{a}$ \\ Jørgen Serup ${ }^{b}$ \\ ${ }^{\mathrm{T} T O O}$ sonix A/S, Hoersholm, Denmark; ${ }^{b}$ Department of Dermatology, Bispebjerg University \\ Hospital, Copenhagen, Denmark
}

\section{Keywords}

High-intensity focused ultrasound · Focused ultrasound - Wart removal · Verruca vulgaris .

Papillomavirus

\begin{abstract}
Therapies of common warts are cumbersome and not very effective. Recurrences are common. A new $20 \mathrm{MHz}$ high-intensity focused ultrasound (HIFU) method is introduced as a new potential treatment modality. With HIFU, selected targets in the epidermis and dermis can be treated with full control of the depth and position of the ultrasound lesion and the energy applied to the target. The treatment can be monitored directly in real-time via an integrated dermoscope in the ultrasound probe. Two warts were treated with 8-10 shoulder-by-shoulder treatment doses, focal depth $1.3 \mathrm{~mm}$, and $1.2 \mathrm{~J} /$ dose. Pretreatment ultrasound B-mode scanning had shown the thickness and depth of the warts. The treated areas developed a dry wound covered by a crust over the next 1-2 days. After 2 weeks the skin was healed, with no wart and no scar. Observation showed no reoccurrence. HIFU has future potential for treatment of common warts and flat warts, and a broad range of skin lesions being logic further candidates for targeted ablative treatment. One single treatment may suffice. It is, therefore, a new modality in dermatology with a large range of indications.
\end{abstract}

\section{Introduction}

Common warts (verruca vulgaris) are a significant burden among dermatology patients. Children are often affected. Social activities can be impaired, and the affected children can be stigmatized in their local environment. Treatments are often practiced as 
repeated application of caustic remedies over a longer period and are thus resource demanding. Routine treatments have limited effectiveness, and autoinoculation and interpersonal spreading is a real risk. More advanced treatment applied to recalcitrant warts includes destructive therapies (e.g., curettage, cryotherapy, carbon dioxide lasers, dye-lasers, or PDT), virucidal agents (e.g., glutaraldehyde), antiproliferative agents (e.g., topical 5-fluorouracil or cidofovir), and immunological therapy (e.g., imiquimod). Common warts are, therefore, despite being positioned as a rather trivial condition, already seen as an object for more expensive instrumental or pharmaceutical therapies in cases when the standard treatment fails $[1,2]$.

Delineation of the extension of a wart is not simple, since the papillomavirus causing the wart is found both horizontally around the wart in the visibly normal skin, and vertically at the bottom of the wart in the dermis. Removal by treatment of the keratotic massive of the core of the wart consisting of dead material is, therefore, bound to be inefficient, unless both the viral border and full depth of the wart are included. Treatment in the deeper part of a wart is, however, difficult. The keratotic center of the wart is a tight barrier toward penetration of chemical caustics and drugs. Sequential horizontal trimming of the wart with a scalpel is often used in the attempt to overcome this problem. However, pain and bleeding often limit the effectiveness of this intervention $[1,2]$.

Good pretreatment diagnosis of the delineation of warts can be obtained from wellestablished methods. The horizontal extension of a wart can be assessed with a dermoscope, while the vertical extent can be visualized by high-frequency ultrasound imaging [3], optical coherence tomography [4], or reflectance confocal microscopy [5].

$20 \mathrm{MHz}$ high-intensity focused ultrasound (HIFU), the method introduced in our case report, is applied as small treatment doses of high-ultrasound energy focused in the dermis and epidermis. HIFU was recently introduced as a new therapeutic option for other dermatological pathologies [6]. While skin is a new field of application, HIFU is far from new in medicine. Noninvasive HIFU operating at lower frequencies from $500 \mathrm{kHz}$ to $3 \mathrm{MHz}$ has been established for treatment of a wide range of internal cancers of major organs, bone metastases, and cerebral pathologies over the last decade. In these treatments, HIFU focal zones are positioned deeply within the body or in the brain with the anatomical location and the chosen focal point guided by MRI scanning or ultrasound imaging. In the focal zone inside the body, reproducible temperatures of about $60-80^{\circ} \mathrm{C}$ are reported [7-10].

In contrast to the low-frequency HIFU devices, the new $20 \mathrm{MHz}$ HIFU system can apply a concentrated ultrasound beam selectively inside the dermis of the skin. Measurements have shown that the temperature in such small focal points rapidly rises to approximately $65^{\circ} \mathrm{C}[6]$. The thermal insult is thereby high enough to kill cells and viruses hosted in the cells or depending on live cell functions [7-10]. With the limited and controlled heating from HIFU compared to, for example, lasers that reach $200-300^{\circ} \mathrm{C}$ in the incident zone, thermal transmission may be less damaging to adjacent tissue that is not a direct treatment object. With different probes, different levels in the skin can be chosen. The duration of the ultrasound pulse and the ultrasound power can be chosen by the operator [6].

Thus, the HIFU method has instrument characteristics offering strictly controlled thermal destruction of warts in both the horizontal and vertical plane. The therapeutic effect can be designed to reach the bottom of the wart. The treatment is applied as a number of separate HIFU treatment doses, which are surveyed real-time by an integrated dermoscope in the probe. The positioning and activation of each HIFU dose are controlled by the operator to systematically cover the entire wart following the precise contour of the individual lesion.

The $20 \mathrm{MHz}$ HIFU used for dermatological applications was studied in experimental laboratory models, animals, and humans. HIFU was concluded safe and relevant for different 
dermatological indications $[6,11]$. Actinic keratosis, basal cell carcinoma, and Kaposi Sarcoma were treated successfully [12]. The case presented is the first application of dermatological $20 \mathrm{MHz}$ HIFU to common warts located on the hand.

\section{Case Presentation}

The subject, a male volunteer aged 44 years, presented 2 warts. One was on the right side of the left ring finger, and the other between the middle and index finger on his left hand. The warts were not treated prior to this study.

Ultrasound scanning prior to HIFU treatment was performed with a $20 \mathrm{MHz}$ Dermascan ${ }^{\circledR}$ C system operated in B-mode (Cortex Technology ApS, Hadsund, Denmark). The integrated software in the ultrasound imaging system was used to measure the depth of the warts in order to guide the selection of HIFU probe [13].

HIFU was applied to the warts using a $20 \mathrm{MHz}$ HIFU device (System ONE-M, TOOsonix A/S, Hoersholm, Denmark) [14]. A probe with nominal focal depth of $1.3 \mathrm{~mm}$ was selected. HIFU energy was administered as separate $1.2 \mathrm{~J}$ doses each of $150 \mathrm{~ms}$ pulse duration at $8 \mathrm{~W}$. Warts were treated with 8 and 10 doses, respectively, administered as shoulder-by-shoulder treatment doses at intervals of approximately $1 \mathrm{~s}$. Standard ultrasound gel was used as coupling medium between probe and skin.

Pretreatment Cross-Sectional Ultrasound Scanning of Lesions to Measure Wart Extension and Thickness

The larger rather circular wart on the finger had a diameter of $3.2 \mathrm{~mm}$ and a depth of 1.2-1.3 mm from the surface of the wart to the estimated deepest point of echolucent tissue. The smaller wart had a diameter of approximately $1.9 \mathrm{~mm}$ and measured $1.0-1.1 \mathrm{~mm}$ in depth. Photos and ultrasound scans are shown in Figure 1.

\section{HIFU Treatment and Immediate Treatment Effects}

The immediate effects of HIFU treatment observed in the treatment field were localized whitening and edema in circular areas of approximately $2 \mathrm{~mm}$ in size following each HIFU dose application. Each dose could easily be observed in real-time via the integrated dermatoscope in the hand piece of the HIFU system, ensuring accurate treatment relative to anatomical target. At end of treatment, the target area showed a blister-like white swelling where the epidermis sometimes had partially separated. In both warts, the area treated remained dry without bleeding. Ultrasound scanning showed an enlarged well-defined echolucent bulk of edema directly under the skin surface in the structure of treatment. Photos and ultrasound scans of HIFU treated lesions directly after HIFU treatment are shown in Figure 2.

Pain level during treatment was scored 6-7 on a 10-point VAS scale. This is relatively high compared to earlier reports of HIFU treatments with the TOOsonix device. This appears to be attributed to the high concentration of sensory nerve endings on the hands. An earlier experiment has, however, shown significant decrease in pain score by self-administering an anesthetic topical (EMLA ${ }^{\circledR}$, AstraZeneca) on the targeted area prior to treatment. In this case, pain was almost eliminated, while treatment response and post-treatment healing remained within the range observed in the subjects [15].

\section{Follow up after Treatment}

After a few days, a dry wound crust was formed on the treated areas. The treated fields gradually healed over 2-3 weeks. The wound crust loosened spontaneously. After 6 weeks, the normal skin structure was almost fully restored, and only a mild inflammatory redness

\section{Karger's}



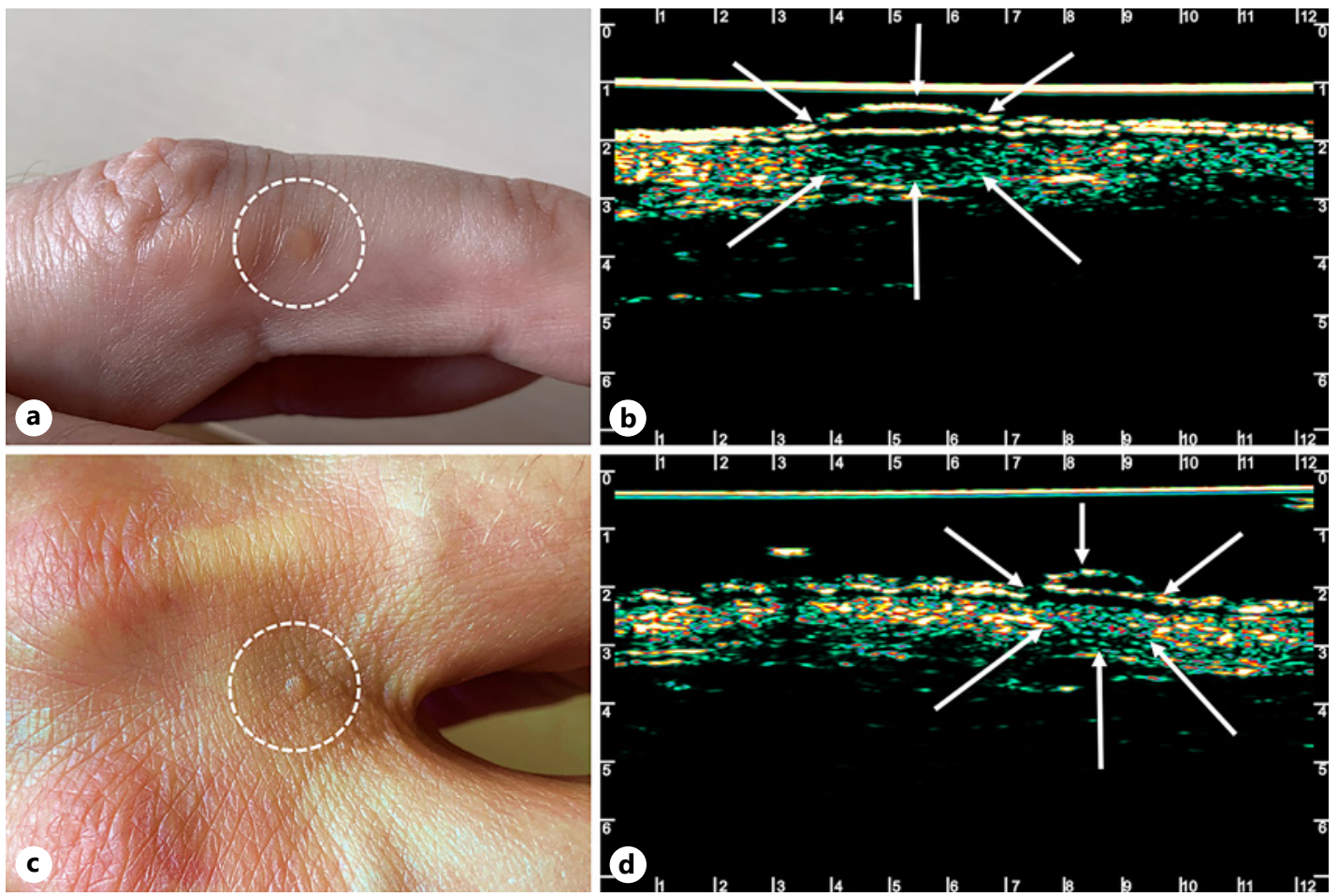

Fig. 1. Photo (a, b) and cross-sectional $20 \mathrm{MHz}$ B-mode ultrasound scans (c, d) of the 2 warts before treatment. Arrows indicate the epidermal surface and the top and margin of the warts.
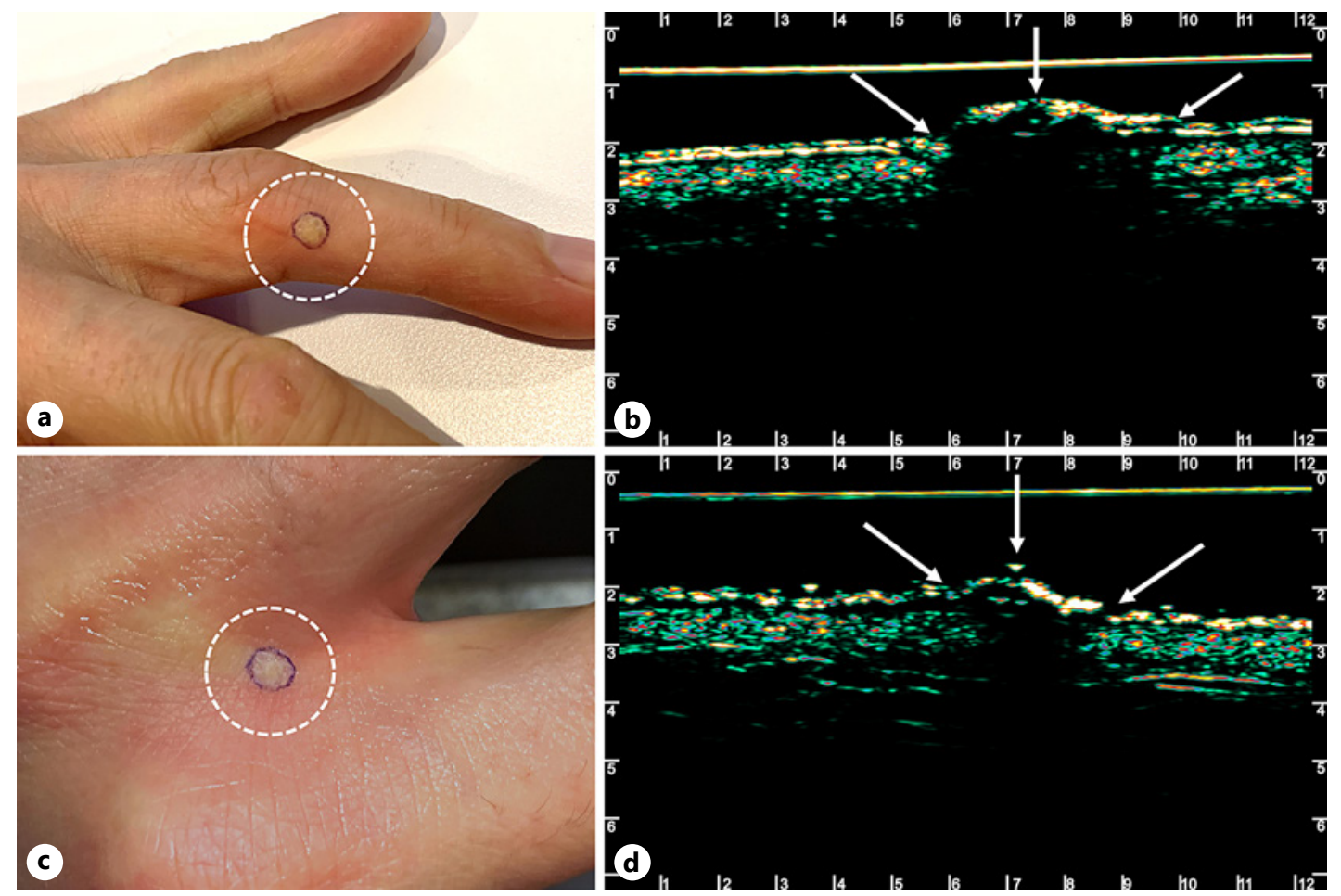

Fig. 2. Photo (a, b) and cross-sectional $20 \mathrm{MHz}$ B-mode ultrasound scans (c, d) of the 2 treated warts directly after HIFU treatment. 

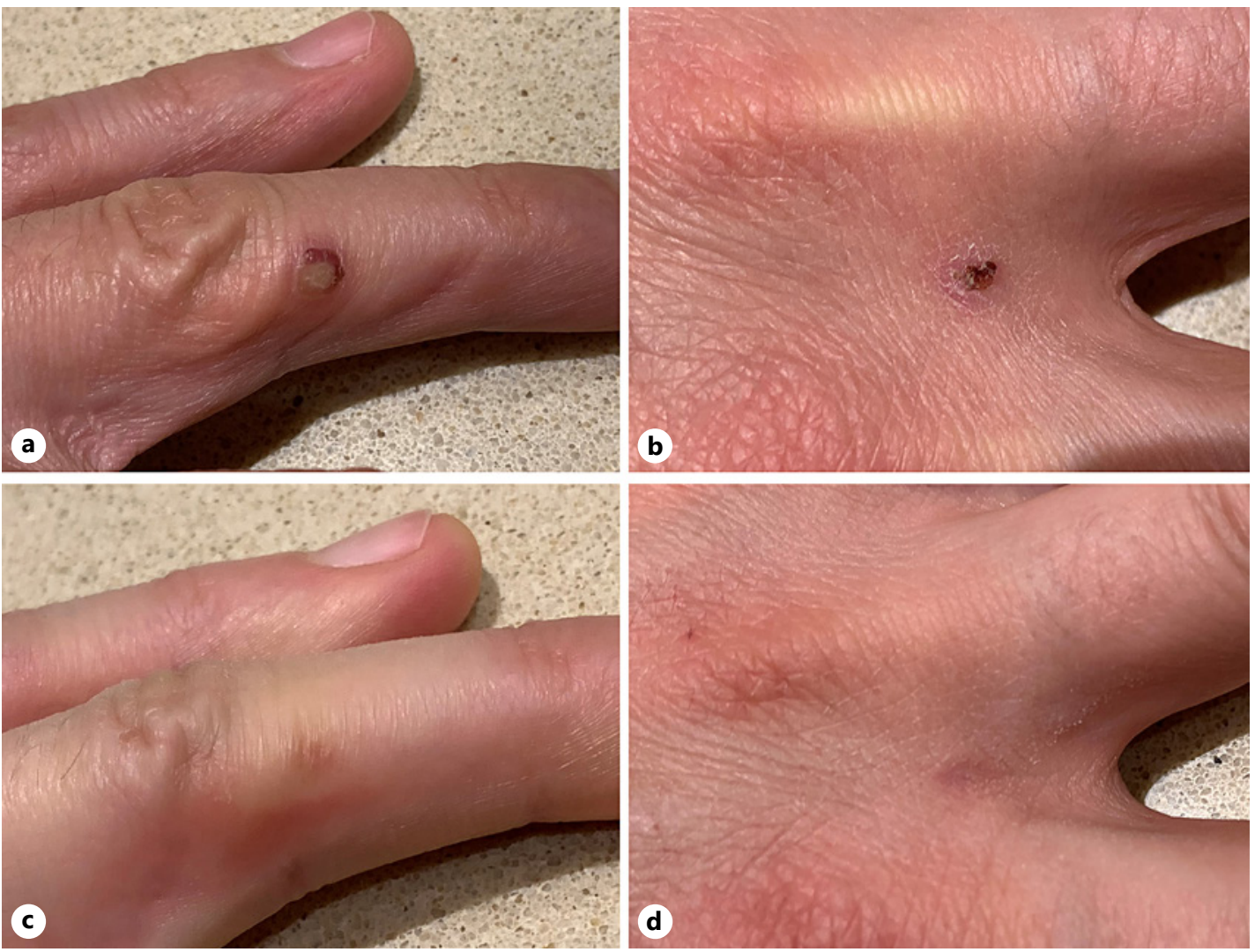

Fig. 3. Photos of treatment areas after 7 days $(\mathbf{a}, \mathbf{b})$ and 6 weeks $(\mathbf{c}, \mathbf{d})$ after HIFU treatment. Both treated areas passed a healing stage of crustation and inflammation. HIFU, high-intensity focused ultrasound.

was visible. After 3 months, the treated fields were completely healed with no reoccurrence of warts, and no scarring or dyspigmentation. Wound healing with crust formation and subsequent regeneration of normal skin is illustrated in Figure 3.

\section{Discussion/Conclusion}

The case illustrates that $20 \mathrm{MHz}$ HIFU can be applied to common warts and produce primary cure with no significant side effects, leaving no scarring. This was achieved with 1 treatment only. It is noteworthy and clearly an advantage relative to other methods, except carbon dioxide lasers, that only one treatment was needed. The level of pain was significant, and however, a lower pain level was found in other studies of other anatomical sites than the hands having many sensory nerves $[12,15]$.

Other treatments producing wart eradication after 1 treatment only includes strong caustics applied to produce direct wart and skin tissue necrosis, carbon dioxide lasers, and possibly electrocautery. These methods are, however, difficult to control with respect to lateral and vertical damage to normal tissue structures, and the risk of scarring is unpredictable since the delineation of the tissue subject to destruction is unclear. The mentioned methods have found no general acceptance, and they are reserved for selected cases. Cryotherapy is widely used for wart treatment in dermatological practice and surely carry less risk of scarring, however, the therapeutic efficacy is variable, and the control of the tissue freeze zone in depth is obscure since the keratotic mass of the wart 
is a thermal insulator and of uncertain thickness. Cryotherapy can furthermore not be guided by real-time dermoscopy as HIFU can.

HIFU has a future potential in the treatment of common warts as well as flat warts since the effect is targeted and highly controlled with limited damage of the surrounding tissue. The method shall be studied in larger materials. The focused ultrasound cone can in principle bypass the keratotic center of a wart and reach the bottom of a wart but is, however, likely that mosaic warts and very thickened warts, including warts on the feet, will require reduction of the keratotic wart mass by knife as a pretreatment. Alternatively, moisturization with an occlusive hydrocolloid bandage for a few days may significantly help deep transmission of the ultrasound energy rendering one-session-only HIFU wart removal possible, even in more advanced keratosis. Further, study shall clarify the effectiveness of pretreatment occlusion, which may easily end up being recommended standard in warts with dry keratosis.

\section{Statement of Ethics}

This study was conducted ethically and in accordance with the World Medical Association Declaration of Helsinki. The subject presented in the study signed a written informed consent regarding participation in treatment and publication of the case.

\section{Conflict of Interest Statement}

Prof. J. Serup is principal clinical investigator and consultant for TOOsonix A/S, Denmark.

\section{Funding Sources}

The treatment presented in this study was performed on equipment funded by TOOsonix A/S, Denmark.

\section{Author Contributions}

All named authors meet the International Committee of Medical Journal Editors (ICMJE) criteria for authorship of the manuscript. Authors take responsibility for the integrity of the work as a whole and grant final approval of the final publication.

\section{References}

1 James WD, Berger TG, Elston DM, editors. Andrews diseases of the skin. 12th ed. Philadelphia: Elsevier; 2016

2 Sterling JC, Gibbs S, Haque Hussain SS, Mohd Mustapa MF, Handfield-Jones SE. British Association of dermatologists' guidelines for the management of cutaneous warts 2014. Br J Dermatol. 2014 Oct;171(4):696-712.

3 Olsen LO, Takiwaki H, Serup J. High frequency ultrasound characterization of normal skin. Skin thickness and echographic density of 22 anatomical sites. Skin Res Technol. 1995 May;1(2):74-80.

4 Friis KBE, Themstrup L, Jemec GBE. Optical coherence tomography in the diagnosis of actinic keratosis: a systematic review. Photodiagnosis Photodyn Ther. 2017 Jun;18:98-104.

5 Rueter L, Ramadori P, Ulrich M, Jung S, Kardorff B, Lademann J. Reflectance confocal microscopy (RCM) for noninvasive examination of nonmelanocytic tumors and virus-associated skin lesions in organ transplant recipients. Skin Res Technol. 2020 May;26(3):376-89. 
6 Bove T, Zawada T, Serup J, Jessen A, Poli M. High-frequency (20-MHz) high-intensity focused ultrasound (HIFU) system for dermal intervention: preclinical evaluation in skin equivalents. Skin Res Technol. 2019; 25(2):217-28.

7 Focused Ultrasound Foundation. State of the field 2020. Available from: http://www.fusfoundation.org/ images/pdf/Focused_Ultrasound_Foundation_2020_State_of_the_Field_Report.pdf Accessed 2020 Dec 9.

8 Ellens NPK, Partanen A. Preclinical MRI-guided focused ultrasound: a review of systems and current practices. IEEE Trans Ultrason Ferroelectr Freq Control. 2017 Jan;64(1):291-305.

9 Barile A, Arrigoni F, Zugaro L, Zappia M, Cazzato RL, Garnon J, et al. Minimally invasive treatments of painful bone lesions: state of the art. Med Oncol. 2017 Apr;34(4):53.

10 Kim M, Jung NY, Park CK, Chang WS, Jung HH, Chang JW. Comparative evaluation of magnetic resonanceguided focused ultrasound surgery for essential tremor. Stereotact Funct Neurosurg. 2017;95(4):279-86.

11 Soegaard S, Aarup V, Serup J, Bove T, Zawada T, Jessen A, et al. High frequency ( $20 \mathrm{MHz}$ ) high intensity focused ultrasound (HIFU) system for dermal intervention: a 12 week local tolerance study in minipigs. Skin Res Technol. 2020;26(2):241-54.

12 Serup J, Bove T, Zawada T, Jessen A, Poli M. High frequency (20 MHz) high-intensity focused ultrasound (HIFU): treatment of actinic keratosis, basal cell carcinoma and Kaposi sarcoma. Skin Res Technol. 2020;26: 824-31.

13 Aps C, Denmark . DermaScan C USB product brochure. Available from: https://cortex.dk/wp-content/ uploads/2019/08/DermaScan-C-USB.pdf.Accessed 2020 Dec 9.

14 TOOsonix A/S Denmark, System ONE-M product brochure. Available from: https://www.toosonix.com/ wp-content/uploads/2020/08/TO0sonix-DS-S02-ver2-web.pdf Accessed 2020 Dec 9.

15 Serup J, Bove T, Zawada T, Jessen A, Poli M. High-frequency (20 MHz) high-intensity focused ultrasound: new ablative method for color-independent tattoo removal in 1-3 sessions. An open-label exploratory study. Skin Res Technol. 2020;26:839-50. 